\title{
Oscillating cylinder in viscous fluid: calculation of flow patterns and forces
}

\author{
Farah Rashid · Magnus Vartdal · John Grue
}

Received: 9 April 2010 / Accepted: 12 July 2010 / Published online: 5 August 2010

(C) The Author(s) 2010. This article is published with open access at Springerlink.com

\begin{abstract}
Laminar and large-eddy-simulation (LES) calculations with the dynamic Smagorinsky model evaluate the flow and force on an oscillating cylinder of diameter $D=2 R$ in otherwise calm fluid, for $\beta=D^{2} / \nu T$ in the range 197-61400 and Keulegan-Carpenter number $K=U_{m} T / D$ in the range 0.5-8 ( $v$ kinematic viscosity, $T$ oscillation period, $U_{m}$ maximal velocity). Calculations resolving the streakline patterns of the Honji instability exemplify the local flow structures in the cylinder boundary layer $(\beta \sim 197-300, K \sim 2)$ but show that the drag and inertia force are not affected by the instability. The present force calculations conform with the classical Stokes-Wang solution for all cases below flow separation corresponding to $K<2$ (with $\beta<61400$ ). The LES calculations of flow separation and vortical flow resolve the flow physics containing a large range of motion scales; it is shown that the energy in the temporal turbulent fluctuations (in fixed points) are resolved. Accurate calculation of the flow separation occurring for $K>2$ has strong implication for the force on the cylinder. Present calculations of the force coefficients for $K$ up to 4 and $\beta=11240$ are in agreement with experiments by Otter (Appl Ocean Res 12:153-155, 1990). Drag coeffients when flow separation occurs are smaller than found in U-tube experiments. Inertia coefficients show strong decline for large $K$ (up to 8) and moderate $\beta=1035$ but is close to unity for $K=4$ and $\beta=11240$. The finest grid has $2.2 \times 10^{6}$ cells, finest radial $\Delta r / R=0.0002$, number of points along the cylinder circumference of $180, \Delta z / R=0.044$ and a time step of $0.0005 T$.
\end{abstract}

Keywords Flow physics · Flow separation · Honji instability $\cdot$ LES calculation · Smagorinsky

\section{Introduction}

Many papers have considered the forces on a long cylinder exposed to oscillatory flow, including, particularly, a group of classical papers by Stokes [1], Wang [2], Honji [3] and Hall [4], experimental papers by e.g. Sarpkaya [5], Otter [6], Chaplin [7], Sarpkaya [8], Johanning et al. [9] and Chaplin and Mouazé [10], and (quite) recent computational papers by $\mathrm{Lu}$ et al. [11], Lu and Ling [12] and Wu et al. [13]. A combined experimental and computational study was given by Dütsch et al. [14]. In the very recent paper by An et al. [15] detailed computations and flow visualizations of Honji vortices below flow separation are provided. The present paper falls into the category of computations. We compute the flow and forces on a long cylinder that oscillates in a fluid otherwise at rest. The

F. Rashid $\cdot$ M. Vartdal $\cdot$ J. Grue $(\varangle)$

Mechanics Division, Department of Mathematics, University of Oslo, Oslo, Norway

e-mail: johng@math.uio.no 
calculations are performed for ranges of the $\beta$-number, Keulegan-Carpenter number $(K)$ and Reynolds number (Re). $\beta$ is up to $61400, K$ up to 8 and $\operatorname{Re}$ up to 45000 .

The Navier-Stokes equations are fully resolved in the laminar flow range, while large-eddy simulation (LES) is employed when the flow is turbulent. LES obtains filtered variants of the velocity and pressure fields, assuming that the filter width captures the motion scales of the inertia range of the turbulent motion [16]. Pioneering works on LES include the original Smagorinsky model of the sub-grid scale (SGS) motion [17,18]. Notable are the development and success of the dynamic Smagorinsky model, as proposed by Germano et al. [19] and further extended by Lilly [20] and Meneveau et al. [21]. Thanks to the steadily increasing computer power, the computational challenge with LES is merely how it best can be performed, rather than how it can be afforded. For recent references on SGS closure and LES, see the book by Pope [22] and the review by Pope [23].

In this paper we describe LES and laminar-flow computations solving the plain or filtered Navier-Stokes equations. The computations are carried out using an extension of the CDP code (v2.5.1), which is an unstructured finite-volume based CFD code developed at Stanford University's Centre for Integrated Turbulence Simulations (CITS) over a period of 10 years. It has been applied to a variety of complex fluid-mechanical problems, including: staggered jet in cross-flow, flow in jet-engine fuel-nozzle rig and full wheel combustor simulation $[24,25]$. The code emphasizes good kinetic energy-conservation properties, which is a necessity for accurate turbulence simulations [26]. Implementation details can be found in [27].

The present effort represents a first step towards a long-term goal, which is to extend the LES code to free-surface motion and the interaction with marine bodies. An important example includes nonlinear wave diffraction from slender vertical cylinders (offshore wind turbines) where a typical diameter may be $5 \mathrm{~m}$, wave amplitude up to $18.5 \mathrm{~m}$ (corresponding to the crest height of the Draupner wave), implying a Keulegan-Carpenter number up to about 20 . The estimate indicates a need to resolve both flow separation and wave breaking at the geometry.

\subsection{Circular cylinder in sinusoidal flow}

Sinusoidally oscillating flow past a fixed circular cylinder has acquired a great deal of attention from fluid dynamicists and engineers because of its theoretical significance and practical importance. The forces acting on a general body are assumed to be the sum of the inertia force, viscous force and drag. The total force can be expressed in terms of Morison's equation which for a long circular cylinder of diameter $D$ takes the form

$F=\frac{1}{4} \pi \rho D^{2} C_{m} \dot{U}+\frac{1}{2} \rho D C_{d}|U| U$.

Here, $\rho$ denotes the density of the fluid and $U=U_{m} \sin (2 \pi t / T)$ the velocity of the flow, with $T$ the period. By (1) the force is represented by the inertia coefficient $C_{m}$ and drag coefficient $C_{d}$. The oscillatory flow can be characterised by two parameters, viz. the Keulegan-Carpenter number defined by $K=U_{m} T / D$, indicating the ratio between the convective and local acceleration, and the Reynolds number defined by $\operatorname{Re}=U_{m} D / v$ where $v$ denotes the kinematic viscosity. Alternatively, Re is replaced by a frequency parameter $\beta$ defined by $\beta=D^{2} / \nu T=\operatorname{Re} / K$. Reference to the Stokes number St in place of the $\beta$-number is sometimes used $(\mathrm{St}=\beta$ ); see below.

Stokes [1] was the first to show that the force on a fixed cylinder exposed to a sinusoidal flow of a viscous fluid is dependent on both $K$ and Re. Wang [2], using the method of inner and outer expansions, extended Stokes' analysis. The Stokes-Wang solution valid for $K \ll 1$, and $\beta \gg 1$, can be reduced to the following form

$$
\begin{aligned}
C_{d} & =\frac{3 \pi^{3}}{2 K}\left[(\pi \beta)^{-\frac{1}{2}}+(\pi \beta)^{-1}-\frac{1}{4}(\pi \beta)^{-\frac{3}{2}}\right], \\
C_{m} & =2+4(\pi \beta)^{-\frac{1}{2}}+(\pi \beta)^{-\frac{3}{2}} .
\end{aligned}
$$

If the cylinder is oscillating in a fluid otherwise at rest, like in the present calculations, the inertia coefficient is expressed by $\hat{C}_{m}=1+4(\pi \beta)^{-\frac{1}{2}}+(\pi \beta)^{-\frac{3}{2}}$.

In an experimental glass-tank facility with fluid otherwise at rest, Honji [3] oscillated back and forth a vertical circular cylinder of height $H=30 \mathrm{~cm}$ and diameter $D$, the latter ranging between 2 and $3.8 \mathrm{~cm}$, such that $H / D \gg 1$. 
He described a three-dimensional instability of the flow appearing in the form of an oscillatory cell pattern in the boundary layer of the oscillating cylinder. The cylinder motion was given by $\frac{1}{2} d(t)=\frac{1}{2} d_{0} \sin (2 \pi f t)$, with $\frac{1}{2} d_{0}$ the amplitude and $f=1 / T$ the frequency. In his original work Honji expressed the appearance of the instability in terms of the non-dimensional amplitude $d_{0} / D$ and Stokes number St $=f D^{2} / \nu$. (The St-number equals the $\beta$-number.) Investigating the range $0.4<d_{0} / D<1.2$ and $70<\mathrm{St}<700$ he identified the domain where the cell pattern appeared: for relatively large St, exceeding about 400, the cell pattern appeared for amplitudes in the range, about $0.4<d_{0} / D<0.7$. For smaller Stokes number, upper and lower bounds of $d_{0} / D$ both increased, however, with the same difference $\left(d_{0} / D\right)_{\max }-\left(d_{0} / D\right)_{\min }$, approximately. The behavior of the cells exhibited locally a mushroom-like streakline pattern. Honji found no regular patters for $d_{0} / D$ above the upper bound where the flow exhibited flow separation and turbulence.

While the present work was in progress, An et al. [15] have, by means of a finite-element method, provided detailed simulations and flow visualizations of the Honji vortices, for $K=2$ and $\beta \sim 100-600$. They were able to identify steady-streaming structures induced by the Honji rolls.

Performing U-tube measurements with a cylinder at rest, but oscillating the water, for $\beta$-numbers in the range between 1035 and 11240, Sarpkaya [5] has maintained that presence of the Honji instability along the cylinder boundary causes a drag force that is higher than predicted by the Stokes-Wang solution (2). This has, however, not been found in experiments with a smooth cylinder oscillating in still water. With $\beta=61400$, Otter [6] showed that the damping forces are close to the Stokes-Wang solution for $K<1.88$ and that the drag coefficient then increases for larger $K$ because of flow separation at the cylinder. In Otter's experiment, theoretical instability value of transition to Taylor-Görtler instability [4] occurred at a Keulegan-Carpenter number of 0.37 . The present numerical calculations of $C_{d}$ and $C_{m}$ agree with Otter's experimental values. Moreover, we find in the regime of the Honji rolls, that their appearance do not change the force coefficient $C_{d}$. This is the same both prior to and after their appearance.

Chaplin and Mouazé [11] have presented a collection of available experimental measurements of the drag coefficient for $100<\beta<2 \times 10^{6}$ and $K$ (about) below the theoretical stability bound of Taylor-Görtler vortices given by Hall [4]. Experiments by Otter [6] and one of the data sets by Johanning [28] exhibit drag coefficients corresponding to the Stokes-Wang solution (JR Chaplin (2010), personal communication). This is also true for parts of the data set of Sarpkaya [5]. An unpublished experimental data set for $\beta=20000$ showed $C_{d} / W \sim 1.03-1.18$ ( $W$ meaning the Stokes-Wang $C_{d}$ ), and computational attempts showed similar forces (JR Chaplin (2010), personal communication). Other experimental, sets included in Chaplin and Mouazé's summary [10] showed drag coefficients twice the Stokes-Wang solution [7,8,28-30]. A problem with the U-tube experiments, where the cylinder is fixed and the water oscillating, is that the input flow may become polluted by boundary-layer effects and turbulence. The scatter in $C_{d}$ at high values of $\beta$ and small $K$ remains an experimental and computational challenge.

The paper is organized as follows. Following the introduction, Sect. 2 describes the numerical code, its implementation, discretization aspects and organization of the computations. In Sect. 3 detailed computations of the Honji instability and the particular mushroom-shaped flow patterns in the cylinder boundary layer are presented. Calculations of the forces, obtaining the inertia and drag coefficients are discussed in Sect. 4 as well as comparison to other force calculations and measurements. A short Sect. 5 discusses the highly local and three-dimensional flow pattern occurring during flow separation, exemplifying also the frequency components of the velocity field. Finally, a conclusion is given in Sect. 6. A description of the particular implementation of the dynamic Smagorinsky model is found in the Appendix.

\section{Numerical approach}

In this investigation the motion is modeled in a frame of reference $O-x, y, z$ fixed to the oscillating cylinder. The $z$-axis coincides with the cylinder axis, $x$-axis is along the oscillatory cylinder motion, $y$-axis in the lateral direction, and the plane $z=0$ midways on the cylinder vertical. Coordinates $(x, y, z)$ or alternatively $\left(x_{1}, x_{2}, x_{3}\right)$ are used. 
For the present calculations a modified version of the CDP 2.5.1 code is employed [27]. It is an unstructured finite-volume node-based code designed for large-eddy simulation (LES) for potentially very large grids using parallel computers. For the present calculations, the incompressible Navier-Stokes version of CDP is modified with a source term in order to incorporate the moving frame of reference of the cylinder. Additionally, for LES, we are using the dynamic Smagorinsky model (see Appendix). This results in the following set of equations

$$
\begin{aligned}
& \frac{\partial \bar{U}_{j}}{\partial x_{j}}=0, \\
& \frac{\mathrm{D} \bar{U}_{j}}{\overline{\mathrm{D}} t}=v \frac{\partial^{2} \bar{U}_{j}}{\partial x_{i} \partial x_{i}}-\frac{\partial t_{i j}^{r}}{\partial x_{i}}-\frac{1}{\rho} \frac{\partial \bar{p}}{\partial x_{j}}+S \delta_{1 j},
\end{aligned}
$$

where $\bar{U}_{j}$ denotes the filtered velocity, $\bar{p}$ the filtered pressure and $t_{i j}^{r}$ the residual-stress tensor. The latter is expressed in terms of the dynamic Smagorinsky model; see the Appendix. Further, $S$ is the source term applying along the $x$-direction given by

$S=\omega U_{m} \cos (2 \pi t / T)$,

where $U_{m}$ is the maximum velocity of the cylinder as defined in the Sect. 1.1 above $(\omega=2 \pi / T)$. All the discretizations of the terms above are found in [27] except for the source term which is discretized according to

$\int_{V_{P}} S \mathrm{~d} V_{P}=S V_{P}$,

where $V_{P}$ is the dual control volume of the node $\mathrm{P}$.

\subsection{Discretization aspects and organization of computations}

We have made fourteen runs, a-k, some with different resolutions (runs b1,2 and j1,2,3), see Tables 1 and 2. The cylinder has radius $R$ and height $H$; this is $6 R$, however, an additional run c of the Honji instability is made with $H=8 R$ (Table 1), as well as a convergence run $\mathrm{k}$ with $H=4 R$ for high values of $\beta$ and $K$ (Table 2). The computational domain is represented by a box of dimensions $(60 R, 20 R, 6 R)$ in $(x, y, z)$, with periodic extensions in all directions.

In runs a,b,c of the Honji instability, for $\beta=197$ and 300 (Table 1), the discretization along the vertical is $\Delta z / R=0.1$. The total number of cells in these runs is $0.8-1.1 \times 10^{6}$. In runs $\mathrm{d}-\mathrm{j} \Delta z / R=0.086$ and run $\mathrm{k}$ $\Delta z / R=0.044$ (Table 2). In the angular direction 128 points are used in runs a-j2,k and 180 in run $\mathrm{j} 3$. In the radial direction the grid cell nearest to the cylinder boundary is $\Delta r / R=0.001$ (runs a-c), $\Delta r / R=0.0004$ ( $\mathrm{d}-\mathrm{g}, \mathrm{i}, \mathrm{j} 1)$ and $\Delta r / R=0.0002(\mathrm{j} 2, \mathrm{j} 3, \mathrm{k})$ (Table 2).

The discretization along the plane $z=0$ is shown in Fig. 1 (runs j2,k). Plot a shows the section of the entire computational domain, with totally $1.5 \times 10^{6}$ sub-volumes; color plot $\mathrm{b}$ shows a magnification of the discretization at the cylinder boundary, including computational results of the velocities and velocity shear in the boundary layer, at $(x, y, z)=(0, R, 0)$, and plot $\mathrm{c}$ shows a stronger magnification. The computation shown in color Fig. $1 \mathrm{~b}$ has parameters $K=4, \beta=11240$. Convergence tests are performed for $K=4, \beta=11240$ using a coarser grid with $1.2 \times 10^{6}$ cells (run j1) and finer one with $2.2 \times 10^{6}$ cells (run j3). In the runs with $K=4$ the time step is $0.0005 T$, in all other runs it is $0.001 T$.

Table 1 Wavelength of Honji mushroom $\frac{\lambda}{D}$

\begin{tabular}{lllllll}
\hline Run & $H$ & $(\beta, K)$ & Present & Honji & Lu and Ling & An et al. \\
\hline a & $6 R$ & $(300,2)$ & 0.5 & 0.6 & 0.59 & $\sim 0.6-0.7$ \\
b1,2 & $6 R$ & $(197,2.07)$ & 0.6 & 0.63 & - & $\sim 0.6-0.7$ \\
c & $8 R$ & $(197,2.07)$ & 0.67 & 0.63 & & $\sim 0.6-0.7$ \\
\hline
\end{tabular}


Table 2 Force coefficients for different values of $\beta$ and $K$

$W$ means Stokes-Wang solution given in (2-3)

\begin{tabular}{llllllc}
\hline Run & $\beta$ & $K$ & $\hat{C}_{m}$ & $\left(\hat{C}_{m}+1\right) / W$ & $C_{d}$ & $C_{d} / W$ \\
\hline $\mathrm{a}$ & 300 & 2.0 & 1.108 & 0.989 & 1.036 & 1.101 \\
$\mathrm{~b} 1,2$ & 197 & 2.07 & 1.135 & 0.989 & 0.868 & 1.104 \\
$\mathrm{~d}$ & 1035 & 2.5 & 1.017 & 0.975 & 0.577 & 1.742 \\
$\mathrm{e}$ & 1035 & 4.86 & 0.856 & 0.896 & 0.919 & 5.391 \\
$\mathrm{f}$ & 1035 & 6.28 & 0.780 & 0.844 & 1.125 & 8.515 \\
$\mathrm{~g}$ & 1035 & 8.0 & 0.566 & 0.753 & 1.335 & 12.89 \\
$\mathrm{~h}$ & 11240 & 1.0 & 1.025 & 1.002 & 0.228 & 0.930 \\
$\mathrm{i}$ & 11240 & 2.0 & 1.013 & 0.996 & 0.184 & 1.498 \\
$\mathrm{j} 1,2,3$ & 11240 & 4.0 & 0.95 & 0.97 & 0.36 & 5.83 \\
$\mathrm{k}$ & 61400 & 0.5 & 1.016 & 1.003 & 0.187 & 0.890 \\
\hline
\end{tabular}

The simulations were carried out at the University of Oslo on a computer with 16 cores operating at $2.13 \mathrm{GHz}$. The simulation time for each case was in the order of 1 week.

\section{Simulations of the Honji rolls}

In the laminar range, secondary streaming is generated in the cylinder's boundary layer. For sufficiently large oscillation amplitude (and Keulegan-Carpenter number), roll pattern becomes generated, superposed on this streaming, as experimentally found by Honji [3]; see Sect. 1.1, and also in recomputations by Lu and Ling [12] and An et al. [15]. Below we shall compare the present computations to those found in $[11,12,15]$. The roll pattern extends vertically along the cylinder boundary and has a maximum in the plane $x=0$. The roll pattern is periodic with period $\frac{1}{2} T$, with maxima at $2 \pi t / T=0, \pi, 2 \pi$ etc. The cycle of the $z$-velocity $w$ is visualized in color Fig. 2 for $K=2.07, \beta=197$ (finest run b in Table 1), showing the rolls for $2 \pi t / T=0, \pi / 4, \pi / 2$. At the latter time the rolls almost disappear before gaining again, keeping the polarity. This computation is well within the domain where the cell pattern appeared in Honji's experiments, with range $0.4<d_{0} / D<1.2$ (corresponding to $1.26<K<3.77$ ) and $70<\beta=\mathrm{St}<700$.

Putting the perturbation velocities in the plane $x=0$ on the form $(v, w)=(\partial \psi / \partial z,-\partial \psi / \partial y)$ the stream function $\psi$ may be integrated, where $\psi=$ constant gives streak lines of the mushroom-shaped instability as visualized in color Fig. 3. The calculations compare well to the experimental measurements by Honji [3] (Fig. 4). The height of the mushroom is $h=0.65 R$; the outer width is $W_{o}=0.62 R$ and inner width $W_{i}=0.4 R$. With a number of five mushrooms appearing along the cylinder length of $6 R$, the wavelength of the instability becomes $\lambda / D=0.6$ in this case. We have carried out four runs simulating the Honji instability as indicated in Table1 by runs a, b1, b2 and c, for $(\beta, K)$ values of $(300,2)$ and $(197,2.07)$. The difference between runs $\mathrm{b} 1$ and $\mathrm{b} 2$ is that two different resolutions are used. In run $\mathrm{c}$ the cylinder of height $8 R$ is used; in this case the wavelength of the instability becomes $\lambda / D=0.67$. Both computations agree with Honji's observation of the wavelength, obtained for two cylinders of diameters 2 and $3.8 \mathrm{~cm}$, both of height $30 \mathrm{~cm}$; see his Fig. 14. The calculations by Lu and Ling [12] show a wavelength of 0.59 when their cylinder becomes tall, while An et al. [15] have found $\lambda / D \sim 0.6-0.7$, see their Fig. 24.

\section{Force calculations}

The pressure and shear force are integrated over the cylinder surface during the simulation time which is up to $23 T$, as illustrated in Fig. 5a for run b1 with $(\beta, K)=(197,2.07)$. After about $t=2 T$ the force is almost repeatable - before this time small transients are present. After a time $t=14 T$ the small instabilities appear at the surface 
(a)

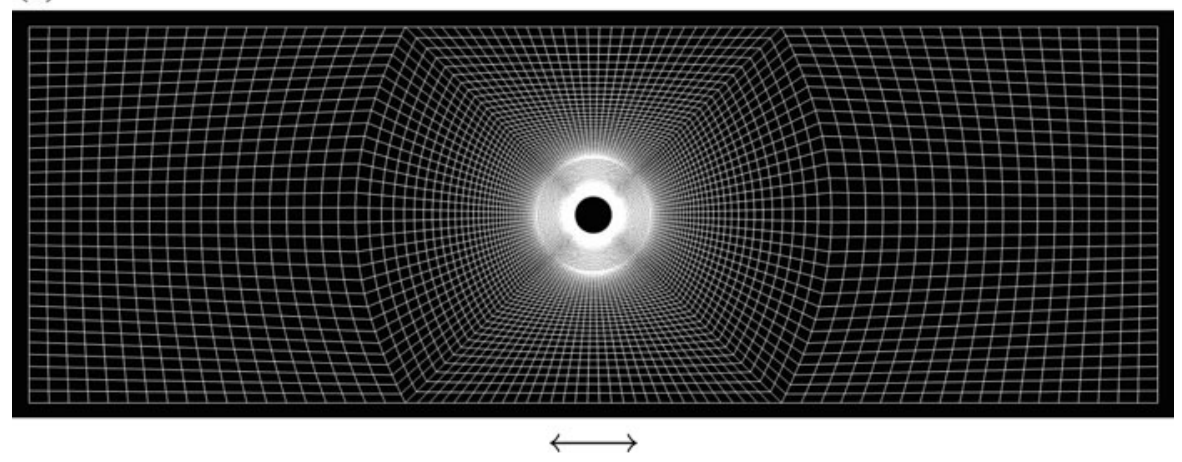

(b)

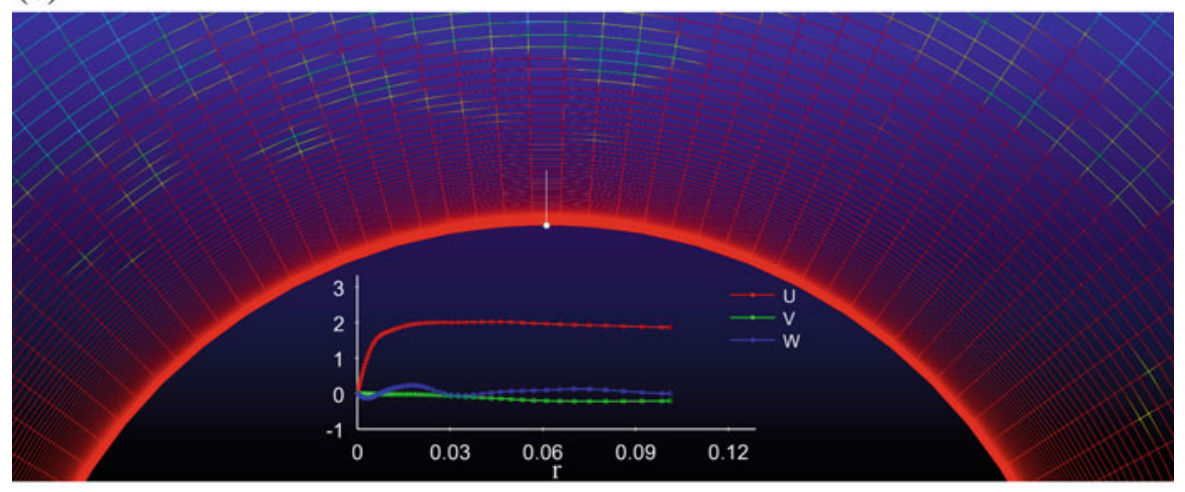

(c)

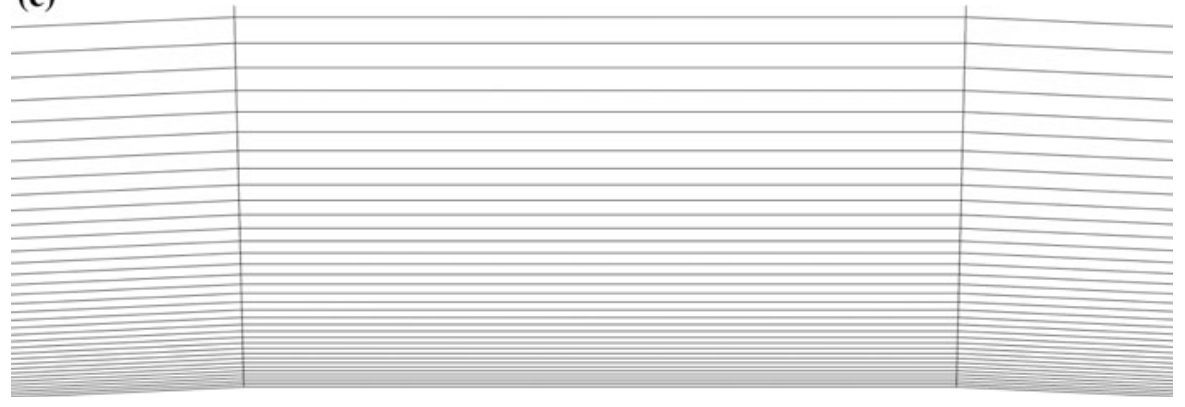

Fig. 1 Example of discretization. Plain cut of mid-section. Total number of cells $1.5 \times 10^{6}$. Arrows indicate cylinder motion. a Complete lateral extension. b Cells at the cylinder in moderate close-up. Also shown are velocities $(u, v, w) / U_{\infty}$ along the radial direction, at the widest part of the cylinder. $\mathbf{c}$ Cells at the cylinder in strong close-up. Innermost cell has $\Delta r / R=0.0002$

of the body; in the present calculations they first appear at the intersection between the cylinder surface and the periodic boundaries at $z= \pm 3 R$. The instabilities grow along the cylinder surface towards $z=0$, from both sides, developing into the rolls and mushroom-shaped instabilities discussed above. An important result of the simulations is that the presence of the Honji instability does not change the drag coefficient. Apart from some initial transients, the value of $C_{d}$ is the same both before and after the instability appears.

Figure $5 \mathrm{~b}$ illustrates the force history for runs $\mathrm{j} 1$ and $\mathrm{j} 2$ for time up to $13 T$, both with $(\beta, K)=(11240,4)$. Run j1 marked with the dashed line has $1.2 \times 10^{6}$ cells, while j2 marked with the solid line has $1.5 \times 10^{6}$ cells. Apart from a small difference during the initial phase, the force histories are identical. Due to the separation taking place at the cylinder, the flow is not entirely symmetric, causing a lateral force acting on the cylinder. This is shown in 
Fig. 2 The cycle of the $z$-velocity $w$ in $x=0$ plane, at the total $z$-direction of the cylinder. $K=2.07$, $\beta=197 . t=0(\mathbf{a})$,

$2 \pi t / T=\pi / 4$ (b) and

$2 \pi t / T=\pi / 2(\mathbf{c})$ (a)

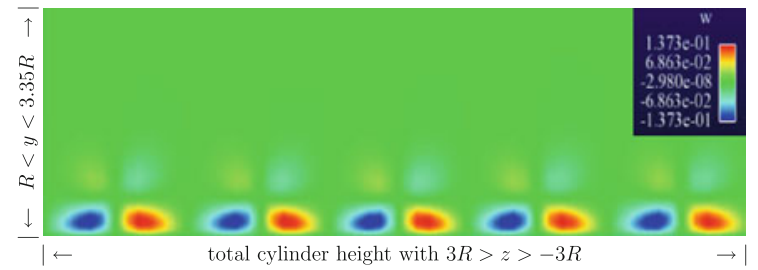

(b)

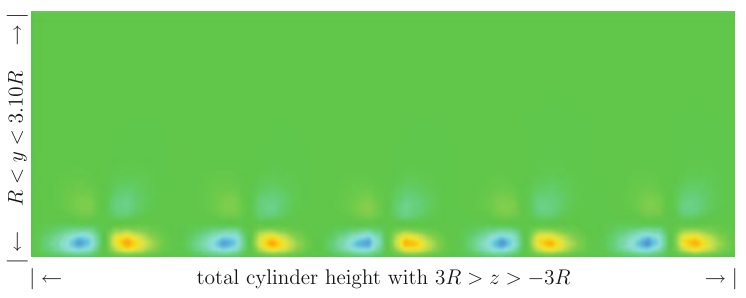

(c)

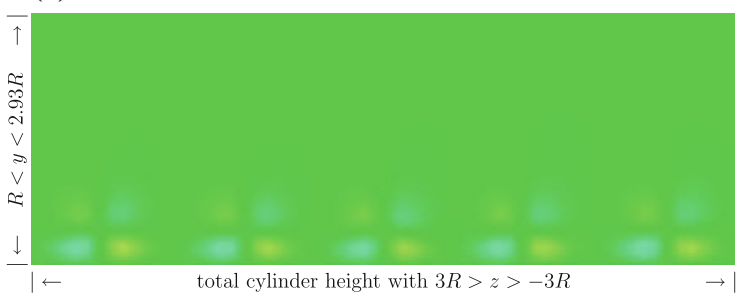

Fig. $5 \mathrm{c}$ for runs $\mathrm{j} 1$ and $\mathrm{j} 2$. It is noted that the transverse force is much smaller than the in-line force. More precisely, $F_{y} / W$ has rms of 0.021 and 0.022 relative to the $\max F_{x} / W$ in runs $\mathrm{j} 1$ and $\mathrm{j} 2$, respectively, for $(\beta, K)=(11240,4)$.

Altogether, we have performed fourteen different computational runs evaluating the flow and force characteristics and thereby extracting the force coefficients according to the Morison equation (1); see Table 2. We have already noted one surprise, namely for the runs in the Honji instability range, where the drag force remained unchanged and close to the Stokes-Wang solution. This has also been true for the other runs, for the larger values of $\beta$, but with $K$ small enough to avoid flow separation $(K<2)$. In this range present computed drag coefficients are close to the Stokes-Wang solution, as visualized in Fig. $5 \mathrm{~d}$.

For the runs when flow separation is dominant, three runs e,f,g, all with $\beta=1035$, and $K=4.86,6.28,8$ show drag coefficients $C_{d}=0.919,1.1125,1.335$ and inertia coefficients $\hat{C}_{m}=0.856,0.780,0.566$, respectively (Re up to 8280), i.e., a strong increase in $C_{d}$ and a strong decline in $\hat{C}_{m}$ (Table 2; Fig. 5e).

In runs $\mathrm{j} 1,2,3$, with the much higher $\beta=11240$ and $K=4$ (and $\operatorname{Re}=45000$ ), the present calculation of the drag coefficient becomes $C_{d}=0.36$. This $C_{d}$-value is in excellent agreement with the measurements of $C_{d}$ performed by Otter [6] of cylinders oscillating in water otherwise at rest, finding $C_{d}=0.31$ for $(\beta, K)=(61400,3.63)$ and $C_{d}=0.44$ for $(\beta, K)=(61400,4.22)$.

In runs $\mathrm{j} 1,2,3$ with $(\beta, K)=(11240,4)$ there is not a big drop in the inertia coefficient, which becomes $\hat{C}_{m}=0.95$.

\subsection{Comparison to other force calculations and measurements}

Results of the drag coefficient, particularly in two publications, are relevant to compare with and discuss. These are the LES simulations by Lu et al. [11] and the U-tube experiments by Sarpkaya [5]. In Lu et al. they simulated 
Fig. 3 'Mushroom' vortices corresponding to the Honji instability in $x=0$ plane, along the total $z$-direction of the cylinder. $K=2.07, \beta=197 . t=0$ (a), $2 \pi t / T=\pi / 4$ (b) and $2 \pi t / T=\pi / 2(\mathbf{c})$ (a)

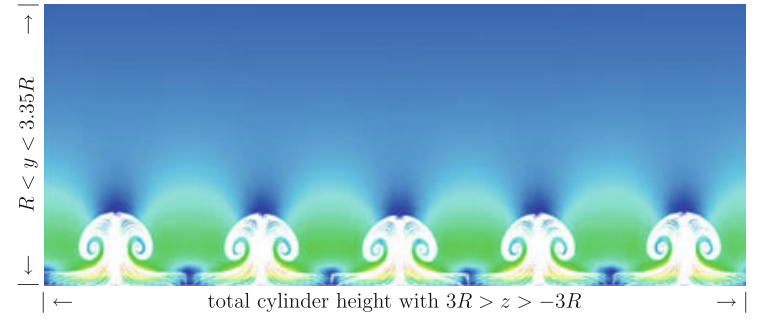

(b)

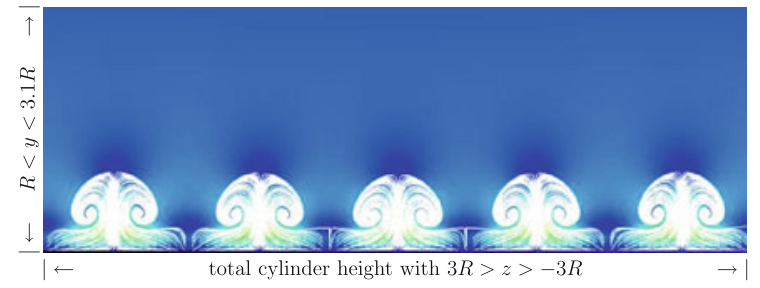

(c)

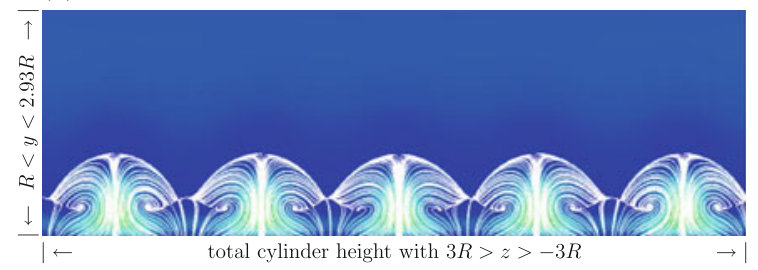

Fig. 4 a Expansion of a single roll in Fig. 3a. Number of grid cells $1.5 \times 10^{6}$. b Close-up view of streaked flow on cylinder. $K=2.07, \beta=197$.

Adapted from [3, Fig. 10]

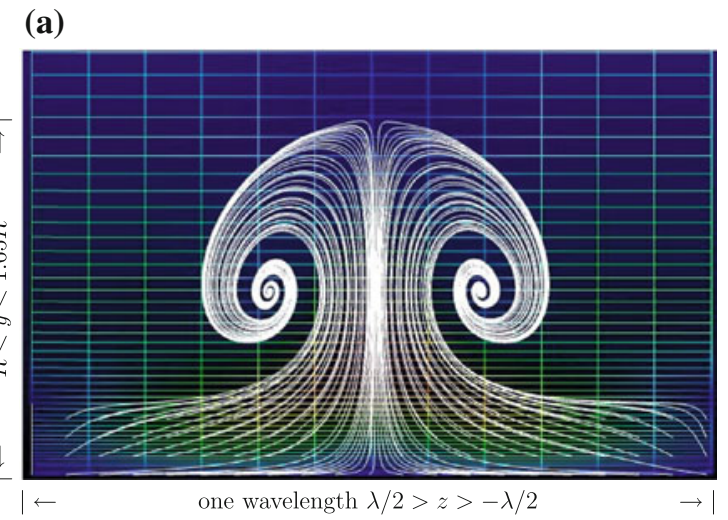

(b)

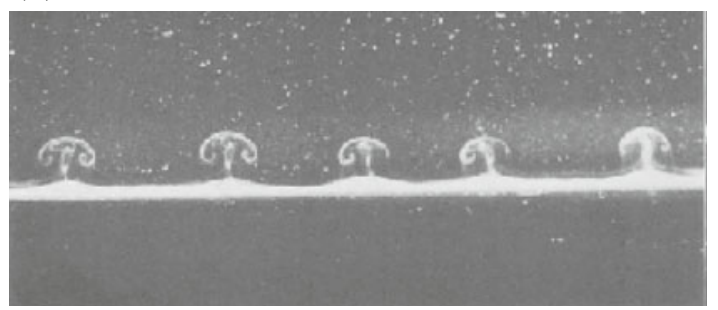



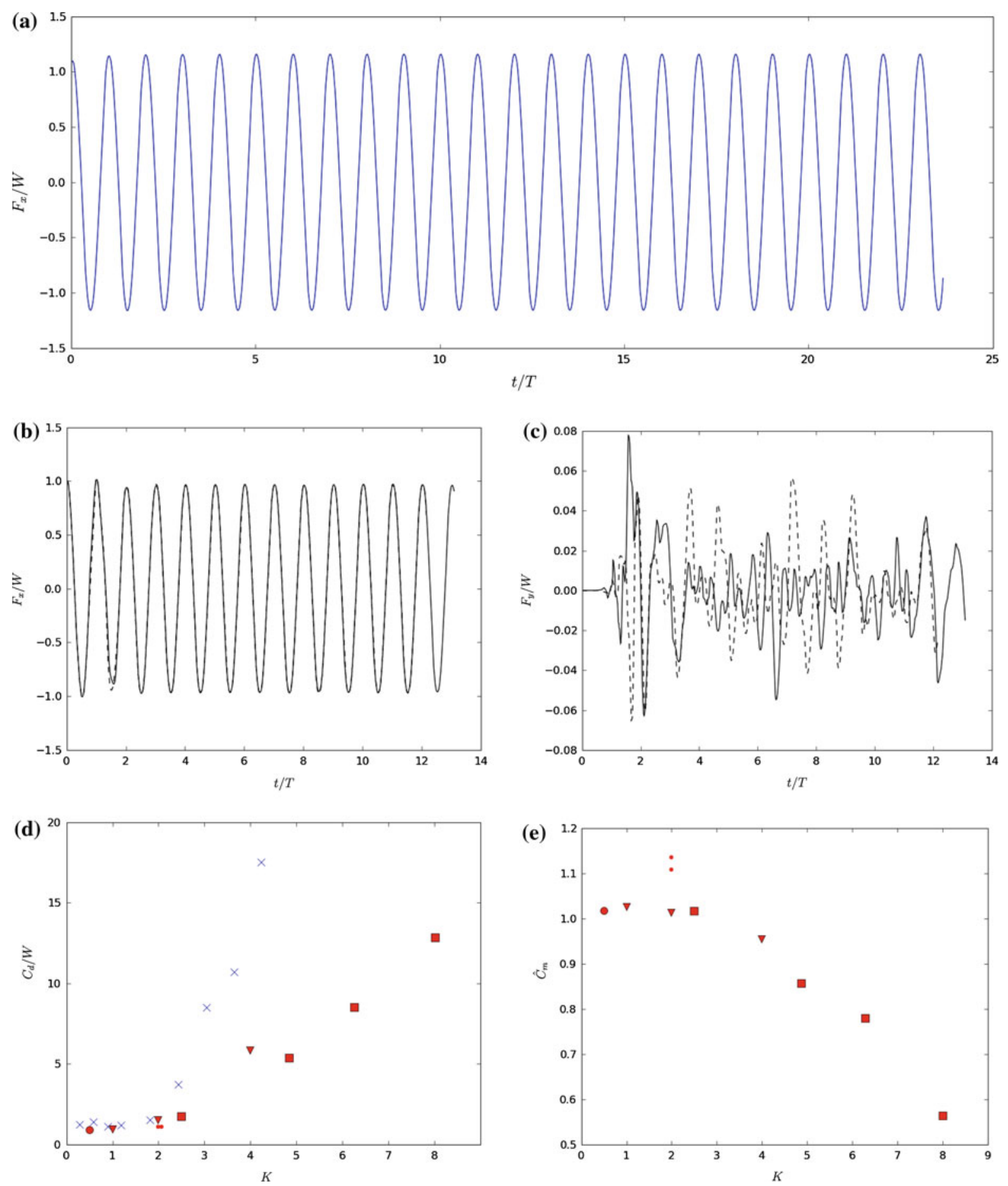

Fig. 5 Force history (along $x$-direction) normalized by Stokes-Wang solution (inertia coefficient) a run b1 with $(\beta, K)=(197,2.07)$; b run $\mathrm{j} 1$ (dashed); run $\mathrm{j} 2$ (solid) with $(\beta, K)=(11240,4)$; c lateral force along $y$-direction in run $\mathrm{j} 1$ (dashed); run $\mathrm{j} 2$ (solid); d drag coefficient divided by Stokes-Wang solution denoted by $W$ versus $K$. Present with $\beta=61400$ (large circle); $\beta=11240$ (triangle); $\beta=1035$ (square); $\beta=300$ and 197 (dot); Otter [6] $\beta=61400$ (crosses); e $\hat{C}_{m}$ versus $K$. Same symbols as in $\mathbf{d}$ 
particularly the data set from Sarpkaya with $\beta=1035$ finding good agreement between the simulations and the measurements. Apart from results fitting to the Stokes-Wang solution, we do not find here the same agreement with the results in Sarpkaya and thus neither with Lu et al. An important disagreement is that the present computations conform to the Stokes-Wang solution until separation takes place.

In the flow-separation range we compare the present computed $C_{d}=0.36$ to Sarkaya's measured $C_{d}=0.5$ for $(\beta, K)=(11240,4)$, his Fig. 3. We also compare present $C_{d}=0.919,1.1125,1.335$ for $K=4.86,6.28,8$, respectively, with $\beta=1035$, to Sarpkaya's measured $C_{d}$ 's in his Fig. 1 . It is evident that his $C_{d}$-values exceed ours by $40 \%$ (i.e., present values multiplied by 1.4 give Sarpkaya's). We note that the inertia coefficients calculated here fit rather well to Sarpkaya's measurements, however.

We note a fundamental difference between U-tube experiments such as those performed by Sarpkaya [5]—oscillating the water while keeping the cylinder fixed, and the experiments by Otter [6]—keeping the water at rest, oscillating the cylinder: it may be speculated if the input oscillatory channel flow gets polluted by turbulence in the former experiments. Present simulations fit well to the experiments by Otter.

We may compare the present computations of the lateral force for $\beta=11240$ and $K=4$ to the computations by $\mathrm{Lu}$ et al. for $\beta=1035$ and $K=3,5$, their Figs. 3 and 7, respectively. While they find a lateral force up to $16 \%$ of $F_{x}$ for $K=3$, and up to and even exceeding $50 \%$ of $F_{x}$ for $K=5$, we find here a rms of $F_{y}$ of only $2 \%$ of $F_{x}$. This is another poor comparison between the present LES calculations and those by Lu et al. We note that in the latter computations the original Smagorinsky model was used with the Smagorinsky constant put equal to 0.1 , as also used in a lot of similar calculations. However, it is evident from several publications that the dynamic Smagorinsky model quite successfully improves the representation of the turbulent motion which is the case when flow separation occurs; see e.g. [23]. Another difference between our and Lu et al. is that here a finer resolution is employed; presently 1.1-2.2 $\times 10^{6}$ grid cells (runs d-k) compared to Lu et al.'s $0.3-1.5 \times 10^{6}$ grid cells.

\section{Local flow structures}

Obtaining the Honji rolls exemplifies the calculation of the local flow structures. The LES calculations at moderate and high $K$ obtain the velocity field during flow separation. There then is strong vortical motion in a large domain surrounding the cylinder. For $K=4$ this domain extends 9 by 4 diameters along the $x$ - and $y$-directions, respectively. For $K=2$ the corresponding extension is 5 by 1 diameters (Fig. 6). The vorticity close to the cylinder is about five times $U_{m} / R$ (Fig. 7), while in the boundary-layer, the vorticity-the velocity shear-is up to 200 times $U_{m} / R$. The boundary-layer thickness in this calculation for $K=4, \beta=11240$ is $0.01 R$ while the grid cell nearest to the wall extends $0.0002 R$ radially (Fig. $1 b$ ).

During flow separation, the velocity and vorticity fields contain a large range of motion scales which are important to represent accurately. The flow separation involving processes on the small scales has to be computed accurately in order to obtain the force well, where the latter is an example of a global quantity. We exemplify the frequency content of the velocity field by computing the time history of $w$-velocity in a fixed points $(x, y, z)=\left(x_{0}, y_{0}, z_{0}\right)$. The auto-correlation function of $w\left(t ; x_{0}, y_{0}, z_{0}\right) / U_{m}$ and its Fourier transform are evaluated as exemplified in Fig. 8. Results are obtained in two points, for $y_{0}=1.02 R$ and $y_{0}=3 R$ with $\left(x_{0}, z_{0}\right)=(0,0)$ is both cases. This clearly show that the energy in the temporal turbulent fluctuations are resolved in the computations.

\section{Conclusions}

Laminar and LES calculations of the flow and force on an oscillating cylinder in otherwise calm fluid have been performed for $\beta$-number ranging from 197 to 61400. The LES code is a modification of CDP, the latter used also for other purposes; it employs the dynamic Smagorinsky model. The computational domain was 30 diameters along the oscillation direction and 10 diameters laterally. The cylinder height $H$ which equals the height of the computational 
Fig. 6 Vorticity $|\nabla \times \mathbf{v}| / U_{\infty} / R$. Cut along mid-section $z=0$. $\beta=11240 . K C=2(\mathbf{a})$ and 4 (b)
Fig. 7 Same as Fig. 6 but

$x=0$ plane, and

$-3 R<z<3 R$,

$R<y<4 R$

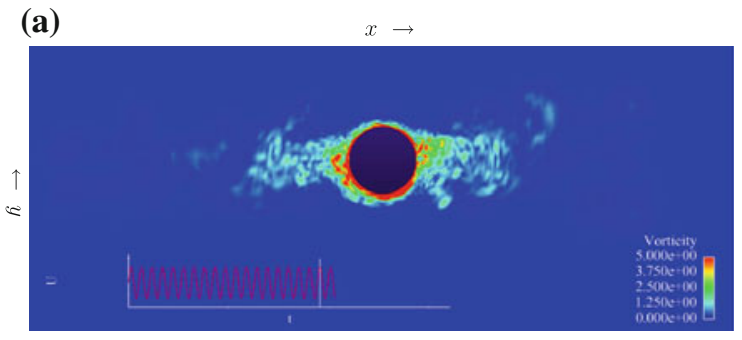

(b)

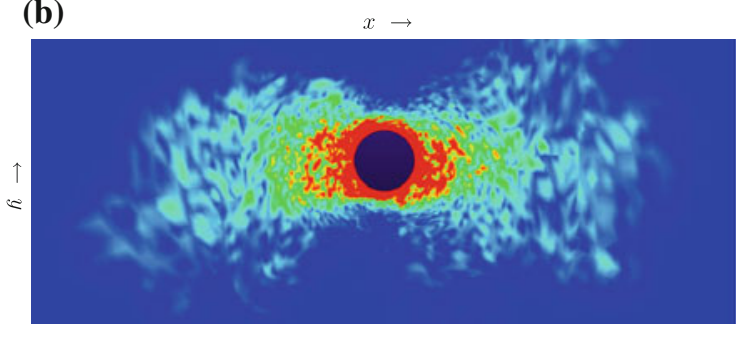

(a)

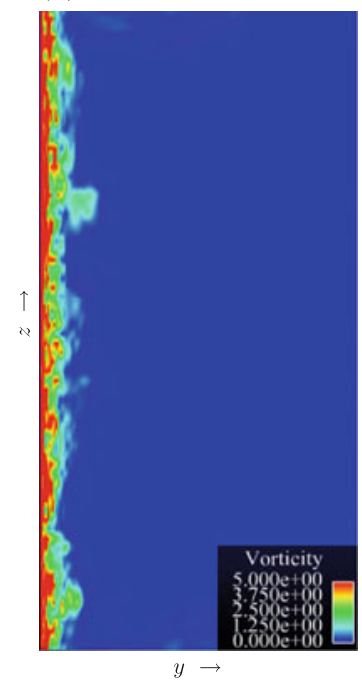

(b)

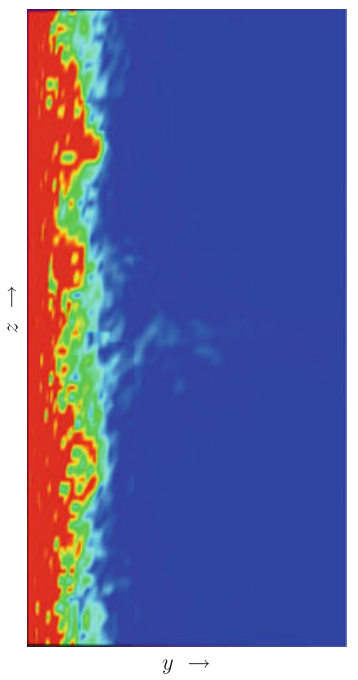

$y \rightarrow$

domain was $6 R$; in two separate runs $H$ is $8 R$ and $4 R$. The computational domain extended periodically in all directions.

We have found as main result that the force coefficients follow (or are very close to) the analytical Stokes-Wang solution for Keulegan-Carpenter number $K$ up to 2 where flow separation occurs. The drag coefficient then departs from the Stokes-Wang solution. Our calculations of the force coefficients are in agreement with the experiments by Otter [6]. He obtained the results for $\beta=61400$ while computations here are performed for a range in $\beta$, up to 61400 , as indicated above. Present calculations of flow separation, performed for $K$ up to 4 and $\beta=11240$, with corresponding $\operatorname{Re}=45000$, exhibit a drag coefficient of $C_{d}=0.36$, comparing well to the experiments by Otter [6], finding $C_{d}=0.31$ for $K=3.63$ and $\beta=61400$, and $C_{d}=0.44$ for $K=4.22$ and $\beta=61400$. Convergence calculations were performed for $K=4$ and $\beta=11240$ as well as for the laminar range. Supplementary computations with smaller $\beta=1035$ and $K=4.86,6.28,8$ (i.e., Re up to 8280) exhibit $C_{d}=0.919,1.1125,1.335$. 

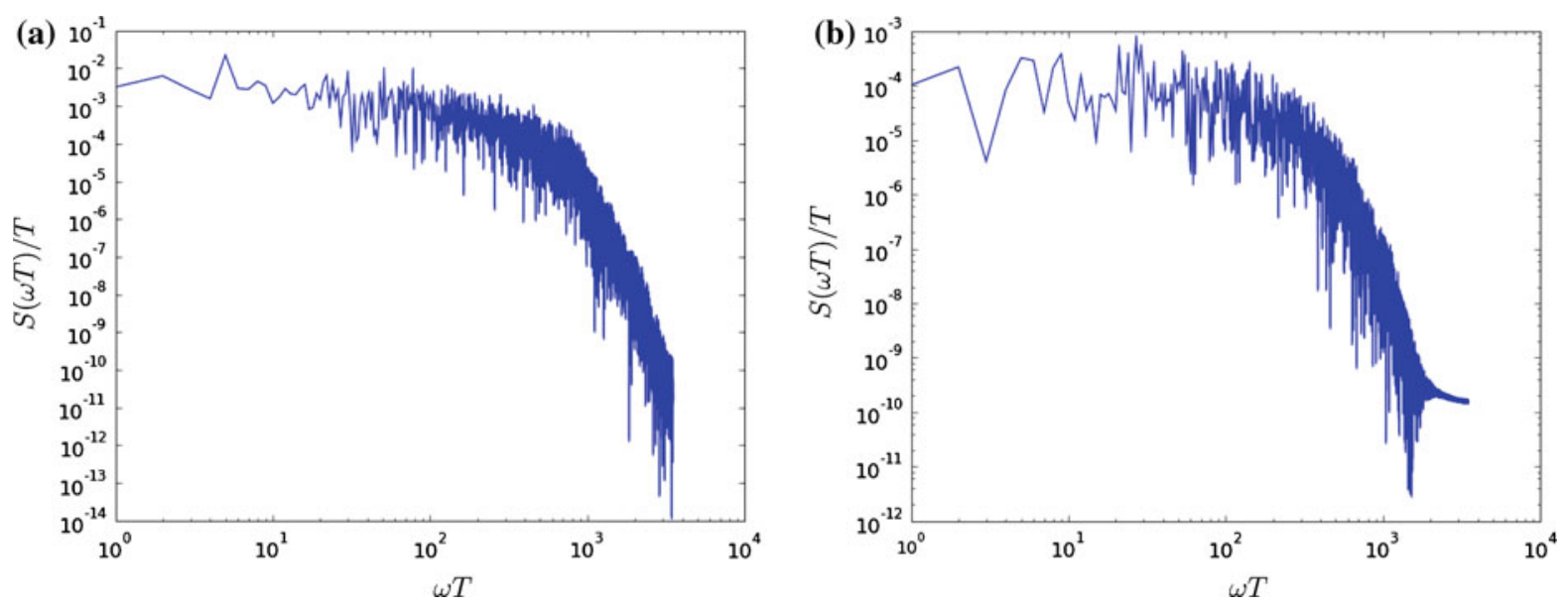

Fig. 8 Temporal spectrum of $w$-velocity in $\left(x_{0}, z_{0}\right)=(0,0)$ and $\mathbf{a} y_{0}=1.02 R, \mathbf{b} y_{0}=3 R$

All present drag coefficient calculations are about $40 \%$ smaller than $C_{d}$ 's measured by Sarpkaya [5] when flow separation occurs. Inertia coefficients calculated here fit rather well to Sarpkaya's measurements, however.

A surprising result of the laminar calculations is that the force coefficients are the same both before and after the appearance of the Honji instability. They agree with the Stokes-Wang solution (they are 10\% higher) but disagree with U-tube experiments obtaining a doubling of the drag coefficient as compared to the Stokes-Wang solution. Particularly Sarpkaya $[5,8]$ has pointed to the Honji instability being the cause of this increase in the drag coefficient. In the present calculations with $(\beta, K)=(197,2.07)$ and $(300,2)$ it takes some time until the Honji instability becomes present; it grows from small instabilities at the intersection between the cylinder geometry and the (periodic) walls at the top and bottom (of the cylinder). The instability then grows along the cylinder side, and becomes fully developed after a number of 14 periods, in the present calculations. Wavelength, height and width of the mushroom-shaped streak patterns induced by the Honji rolls fit well to the original experiments [3]. The periodic appearance and disappearance of the rolls are visualized. Fine and moderate grid resolutions show the same streak pattern.

Several experimental sets at high $\beta$ and $K$ below Hall's stability limit exhibit drag coefficients corresponding to the Stokes-Wang solution. These include [6] and one of the data sets by Johanning [28]. The same has been found in an unpublished experimental data set for $\beta=20000$ (JR Chaplin (2010), personal communication), and also in other computational attempts than those presented here (JR Chaplin (2010), personal communication). However, Otter's experiments [6] conform also to the Stokes-Wang solution well beyond the stability limit, up to where flow separation occurs, at $K=2$. These experiments-physical and numerical-conform with the present calculations. Other experimental sets at high $\beta$ and below the stability limit [7,8,28-30] show drag coefficients twice the StokesWang solution; see the summary by Chaplin and Mouazé's [10]. We note that a problem with the (physical) U-tube experiments, where the cylinder is fixed and the water oscillating, is that the input flow may become polluted by boundary-layer effects and turbulence. The scatter in $C_{d}$ at high values of $\beta$ and small $K$ remains an experimental and computational challenge.

Calculations of the Honji rolls exemplify the local flow structures. The present calculations show that the pattern has no consequence for the force on the cylinder, however. The LES calculations of flow separation and vortical flow resolve the flow physics at small scale. The calculations exhibit velocity and vorticity fields containing a large range of motion scales. Evaluation of the frequency contents of the velocity field shows that the energy of the temporal turbulent fluctuations (in fixed points) is resolved in the computations. It is evidenced that the flow separation occurring for $K>2$ has strong implication for the force on the cylinder. An accurate numerical representation of the flow physics during the separation is needed in order to accurately calculate the force. This implies a relevant modeling of the scales of the flow separation process, that also is three-dimensional. 
The finest grid used had a number of $2.2 \times 10^{6}$ cells; it had a finest radial $\Delta r / R=0.0002$, number of points along the cylinder circumference of $180, \Delta z / R=0.044$ and time step of $0.0005 T$, for the case $K=4, \beta=11240$. The simulations presented here were carried out on a computer with 16 cores operating at $2.13 \mathrm{GHz}$. The simulation time for each case was of the order of 1 week.

Acknowledgements This paper is presented in memory of Professor E. O. Tuck who, with his brilliant analytical approach to a large variety of problems, particularly has inspired the international environments of Applied Mathematics and Hydrodynamics. This research was funded by the Research Council of Norway through NFR 191204/V30.

Open Access This article is distributed under the terms of the Creative Commons Attribution Noncommercial License which permits any noncommercial use, distribution, and reproduction in any medium, provided the original author(s) and source are credited.

\section{Appendix: The dynamic Smagorinsky model}

In this section we describe how the dynamic Smagorinsky model (DSM) is implemented in the present numerical code. The theoretical descriptions of the DSM is given in e.g. [19,20] and [22].

As our starting point for the description of the dynamic Smagorinsky model we consider the plain Smagorinsky model in which the deviatoric part of the residual stress tensor is approximated by

$t_{i j}^{r}=-2 c_{S} \bar{\Delta}^{2} \bar{S} \bar{S}_{i j}$

where $\bar{\Delta}$ is the filterwidth $\bar{S}=\left(2 \bar{S}_{i j} \bar{S}_{i j}\right)^{1 / 2}$ is the characteristic rate of strain, $\bar{S}_{i j}$ is the filtered rate of strain and $c_{S}$ is the square of the Smagorinsky constant. In the Smagorinsky model $c_{S}$ is, as the name implies, a constant, e.g. in the work of Lu et al. [11] the Smagorinsky constant is put equal to 0.1, as also used in a lot of similar calculations. In general, however, we must expect that this is not the case; $c_{S}$ must be viewed as a function of both space and time.

The main idea of the DSM is to apply a second (test) filter, of width $\hat{\Delta}$, to extract the information about the spatial variability of $c_{S}$. Usually $\hat{\Delta} \simeq 2 \bar{\Delta}$. Applying the test filter to the filtered $\mathrm{N}-\mathrm{S}$ equations results in a similar equation, but with a different residual stress expression,

$T_{i j}=\widehat{\overline{U_{i} U_{j}}}-\hat{U_{i}} \hat{U_{j}}$,

We approximate the deviatoric part of $T_{i j}$ in the Smagorinsky manner so that we get,

$T_{i j}^{r}=-2 c_{S} \hat{\bar{\Delta}}^{2} \hat{\bar{S}} \hat{\bar{S}}_{i j}$,

If we now consider the difference between (10) and the test filtered (8) we obtain the following if we, quite inconsistently, assume $c_{S}$ to be uniform [22],

$L_{i j}^{S}=T_{i j}^{r}-\widehat{t}_{i j}^{r}=c_{S}\left(2 \bar{\Delta}^{2} \widehat{\bar{S}}_{i j}-2 \hat{\bar{\Delta}}^{2} \hat{\bar{S}} \hat{\bar{S}}_{i j}\right)=c_{S} M_{i j}$

Here $L_{i j}^{S}$ is an approximation to the deviatoric part of the Leonard stresses, where the Leonard stresses are the difference between the two residual stress tensors,

$L_{i j}=T_{i j}-\widehat{t_{i j}}=\widehat{\overline{U_{i}} \overline{U_{j}}}-\hat{U_{i}} \hat{U_{j}}$.

As we can see the terms in (12) are known in terms of the filtered velocities and can thus be computed. This means that we can calculate a value of $c_{S}$ that minimizes the difference between $L_{i j}^{S}$ and the deviatoric part of $L_{i j}$. This is done by minimizing the mean-square error [20] according to,

$c_{S}=\frac{M_{i j} L_{i j}}{M_{k l} M_{k l}}$ 
In the present numerical code the eddy viscosity $v_{T}=c_{S} \bar{\Delta}^{2} \bar{S}$ is computed using the filtered velocity field from the previous timestep. The coefficient $c_{S}$ is computed according to

$c_{S} \bar{\Delta}^{2}=\frac{\widehat{M_{i j} L_{i j}}}{\widehat{M_{k l} M_{k l}}}$,

where $L_{i j}$ is as given above. In this particular application $M_{i j}$ is modified into

$M_{i j}=2\left(\widehat{\bar{S}}_{i j}-\hat{\bar{\Delta}}^{2} / \bar{\Delta}^{2} \hat{\bar{S}} \hat{\bar{S}}_{i j}\right)$.

The local averaging obtained by the filtering in (14) is necessary for stability [19]. As an additional stabilising step all negative values of eddy viscosity are set to zero.

Finally, to complete the description of the model the filters and their widths need to be specified. For the $\bar{\Delta}$ width filter the filtering is implicit and the filterwidth is taken to be proportional to the mesh spacing, i.e., $\bar{\Delta}=(V)^{1 / 3}$, where $V$ is the control volume. The $\hat{\Delta}$ filter is a weighted average of the value at the node and its neighbours. The filter width ratio is taken to be $\hat{\bar{\Delta}} / \bar{\Delta}=\sqrt{6}$.

\section{References}

1. Stokes GG (1851) On the effect of the internal friction of fluids on the motion of pendulums. Trans Camb Philos Soc 9:8-106

2. Wang C-Y (1968) On high-frequency oscillatory viscous flows. J Fluid Mech 32:55-68

3. Honji H (1981) Streaked flow around an oscillating circular cylinder. J Fluid Mech 107:509-520

4. Hall P (1984) On the stability of the unsteady boundary layer on a cylinder oscillating transversely in a viscous fluid. J Fluid Mech 146:347-367

5. Sarpkaya T (1986) Force on a circular cylinder in viscous oscillatory flow at low Keulegan-Carpenter numbers. J Fluid Mech 165:61-71

6. Otter A (1990) Damping forces on a cylinder oscillating in a viscous fluid. Appl Ocean Res 12:153-155

7. Chaplin JR (2000) Hydrodynamic damping of a cylinder at $\beta \approx 10^{6}$. J Fluids Struct 14:1101-1117

8. Sarpkaya T (2001) Hydrodynamic damping and quasi-coherent structures at large Stokes numbers. J Fluids Struct 15:909-928

9. Johanning L, Bearman PW, Graham JMR (2001) Hydrodynamic damping of a large scale surface piercing circular cylinder in planar oscillatory motion. J Fluids Struct 15:891-908

10. Chaplin JR, Mouazé D (2004) Disturbed laminar flow over an oscillating cylinder. In: XXI international congress on theoretical and applied mechanics, 15-21 Aug 2004, Warsaw, Poland. http://fluid.ippt.gov.pl/ictam04/CD_ICTAM04/FSM4/12557/FSM4_12557. pdf

11. Lu X, Dalton C, Zhang J (1997) Application of large eddy simulation to an oscillating flow past a circular cylinder. J Fluids Eng 119:519-525

12. Lu X-Y, Ling GC (2003) Three-dimensional instability of an oscillating viscous flow past a circular cylinder. Appl Math Mech (English edition) 24:791-800

13. Wu J-Z, Lu X-Y, Zhuang L-X (2007) Integral forces acting on a body due to local flow structures. J Fluid Mech 576:265-286

14. Dütsch H, Durst F, Becker S, Lienhart H (1998) Low-Reynolds-number flow around an oscillating circular cylinder at low Keulegan-Carpenter numbers. J Fluid Mech 360:249-271

15. An H, Cheng L, Zhao M (2010) Direct numerical simulation of oscillatory flow around a circular cylinder at low KC number. J Fluid Mech (in press)

16. Leonard A (1975) Energy cascade in large-eddy simulations of turbulent flows. Adv Geophys 18(1):237-248

17. Smagorinsky J (1963) General circulation experiments with the primitive equations. Mon Weather Rev 91(3):99

18. Lilly DK (1967) The representation of small-scale turbulence in numerical simulation experiments. In: Goldstine HH (ed) Proceedings of IBM scientific computing symposium on environmental sciences. IBM, Yorktown Heights, NY, pp 195-210

19. Germano M, Piomelli U, Moin P, William HC (1991) A dynamic subgrid-scale eddy viscosity model. Phys Fluids A Fluid Dyn 3:1760-1765

20. Lilly DK (1992) A proposed modification of the Germano subgrid-scale closure method. Phys Fluids A Fluid Dyn 4:633-635

21. Meneveau C, Lund TS, Cabot WH (1996) A Lagrangian dynamic subgrid-scale model of turbulence. J Fluid Mech 319:353-385

22. Pope SB (2000) Turbulent flows. Cambridge University Press, Cambridge

23. Pope SB (2004) Ten questions concerning the large-eddy simulation of turbulent flows. New J Phys 6:35. doi:10.1088/1367-2630/ $6 / 1 / 035$

24. CITS (2004) Annual technical report. Center for Integrated Turbulence Simulations, Stanford. http://www.stanford.edu/group/cits/ pdf/reports/cits2004.pdf 
25. CITS (2005) Annual technical report. Center for Integrated Turbulence Simulations, Stanford. http://www.stanford.edu/group/cits/ pdf/reports/cits2005.pdf

26. Ham F, Iaccarino G (2004) Energy conservation in collocated discretization schemes on unstructured meshes. Centre for Turbulence Research, Stanford. http://ctr.stanford.edu/ResBriefs04/ham_iaccarino.pdf

27. Ham F, Mattasson K, Iaccarino G (2006) Accurate and stable finite volume operators for unstructured flow solvers. Centre for Turbulence Research, Stanford. Annual Research Briefs. http://ctr.stanford.edu/ResBriefs06/19_ham1.pdf

28. Johanning L (2003) PhD thesis. Imperial College, London

29. Bearman PW, Russel MP (1996) Measurements of hydrodynamic damping of bluff bodies with application to the prediction of viscous damping of TLP hulls. In: 21st symposium on naval hydrodynamics, Trondheim, Norway, pp 61-73

30. Chaplin JR, Subbiah K (1998) Hydrodynamic damping of a cylinder in still water and in a transverse current. Appl Ocean Res 20:251-259 